\title{
Needle EMG of the tongue: motor unit action potential versus peak ratio analysis in limb and bulbar onset amyotrophic lateral sclerosis
}

\author{
J Finsterer, A Fuglsang-Frederiksen, B Mamoli
}

\begin{abstract}
Objectives-to find out if conventional and automatic needle EMG of the tongue can be helpful in the diagnosis and differentiation of limb and bulbar onset amyotrophic lateral sclerosis.

Methods-Motor unit action potential (MUAP) analysis and peak ratio interference pattern analysis were performed in the right genioglossus muscle of 30 healthy subjects aged 30-81 years, 10 patients aged 49-73 years with limb onset amyotrophic lateral sclerosis, and eight patients aged 52-75 years with bulbar onset amyotrophic lateral sclerosis. Electrical activity was sampled via standard concentric needle electrodes with a commercially available EMG recorder.
\end{abstract}

Results-Normal mean (2SD) MUAP duration was $6.6(1.5) \mathrm{ms}$. Normal mean (2SD) MUAP amplitude was $224(97.4) \mu \mathrm{V}$. Normal mean (2SD) peak ratio (PR), turns/second (T/s), amplitude/turn (A/T), and time intervals (TI1, TI2, TI3) were 1.68 (0.56), 732 (303.9), 446 (180.3) $\mu \mathrm{V}, 2.62$ $(0.34), 2.31(0.14)$, and $1.01(0.50)$ respectively. Mean MUAP duration and amplitude were significantly increased in limb onset $(P=0.0001$ and $P=0.013)$ and bulbar onset amyotrophic lateral sclerosis $(\mathbf{P}=0.0001$ and $\mathbf{P}=0.017)$. Peak ratio indices stayed unchanged in limb onset amyotrophic lateral sclerosis but were significantly decreased (PR, T/s, A/T, TI1, and TI2) or increased (TI3) in bulbar onset disease. The sensitivity of the MUAP analysis was $70 \%$ in $\operatorname{limb}$ and $75 \%$ in bulbar onset amyotrophic lateral sclerosis. The sensitivity of the peak ratio interference pattern analysis was $20 \%$ in limb and $100 \%$ in bulbar onset amyotrophic lateral sclerosis. Subclinical involvement of the tongue was found in $20 \%$ of the patients with limb onset amyotrophic lateral sclerosis and could be more accurately assessed with MUAP analysis than with automatic EMG.

Conclusions-both conventional and automatic needle EMG of the tongue are valuable electrophysiological devices to assess the clinical and subclinical involvement of the tongue in patients with limb and bulbar onset amyotrophic lateral sclerosis. and in revised form

26 March 1997

Accepted 27 March 1997
Keywords: tongue electromyography; amyotrophic lateral sclerosis; peak ratio; Norris score; Frenchay aphasia test

Among various neuromuscular disorders, the tongue is most often involved in amyotrophic lateral sclerosis. To improve the accuracy of the diagnosis of amyotrophic lateral sclerosis, information about the electrical activity of the tongue could be helpful. Unfortunately, little information concerning needle EMG of the tongue is available for healthy subjects and patients with amyotrophic lateral sclerosis. ${ }^{12}$ Particularly, different EMG techniques have not been applied to the tongue and compared with each other. The aim of this study was thus (1) to find out if MUAP analysis and peak ratio interference pattern analysis of the tongue are different between healthy subjects and patients with limb and bulbar onset amyotrophic lateral sclerosis and (2) to compare the sensitivity and specificity of MUAP versus peak ratio interference pattern analysis of the tongue in patients with amyotrophic lateral sclerosis with limb onset and bulbar onset.

\section{Methods}

SUBJECTS

We investigated 30 healthy subjects (11 women, 19 men), aged $30-81$ years, 10 patients aged 49-73 years with limb onset amyotrophic lateral sclerosis (six women, four men), and eight patients aged 52-75 years with bulbar onset amyotrophic lateral sclerosis (three women, five men) (table 1). The diagnosis of amyotrophic lateral sclerosis was established by clinical, electroneurographical, and EMG (from muscles other than the tongue) examinations according to the El Escorial criteria. ${ }^{3}$ All healthy subjects and patients gave consent after full explanation of the procedure. The study was approved by the institutional review board.

\section{PROCEDURE}

MUAP analysis and peak ratio interference pattern analysis were carried out in the right genioglossus muscle, one after the other, with the subjects sitting upright. After standardised local anaesthesia with a lidocaine spray and waiting for one minute, the needle was inserted into the right edge of the anterior third of the tongue to a depth of about $20 \mathrm{~mm}$. Subjects were told to anteflect their head, not to swallow, and to leave their mouth open during the procedure. The saliva running out of the open mouth was wiped off by the patient. The 
Table 1 Patient characteristics and their clinical and electrophysiological findings

\begin{tabular}{|c|c|c|c|c|c|c|c|c|c|}
\hline Patient No & Sex & Age (y) & UMNS & $B S$ & $\begin{array}{l}\text { Norris score } \\
(\%)\end{array}$ & $\begin{array}{l}\text { Frenchay test } \\
(\%)\end{array}$ & $S A$ & $\begin{array}{l}\text { MUAP } \\
\text { analysis }\end{array}$ & $\begin{array}{l}\text { Peak ratio } \\
\text { analysis }\end{array}$ \\
\hline \multicolumn{10}{|l|}{ Limb onset } \\
\hline 1 & $\mathrm{~F}$ & 58 & + & + & 55 & 88 & SP & NG & NG \\
\hline 2 & $M$ & 73 & + & - & 54 & 97 & SP & NG & $\mathrm{N}$ \\
\hline 3 & $\mathrm{~F}$ & 64 & - & + & 61 & 95 & $\mathrm{U}$ & NG & $\mathrm{N}$ \\
\hline 4 & $\mathrm{~F}$ & 52 & + & + & 82 & 86 & SP & NG & $\mathrm{N}$ \\
\hline 5 & M & 70 & + & - & 70 & 91 & $\mathrm{U}$ & $\mathrm{N}$ & $\mathrm{N}$ \\
\hline 6 & $\mathrm{~F}$ & 49 & + & - & 77 & 98 & $\mathrm{U}$ & $\mathrm{N}$ & $\mathrm{N}$ \\
\hline 7 & $M$ & 64 & + & - & 76 & 89 & SP & $\mathrm{N}$ & $\mathrm{N}$ \\
\hline 8 & $\mathrm{~F}$ & 52 & + & - & 85 & 100 & SP & NG & $\mathrm{N}$ \\
\hline 9 & $\mathrm{~F}$ & 69 & + & + & 45 & 73 & SP & NG & $\mathrm{N}$ \\
\hline 10 & M & 55 & + & + & 83 & 88 & $\mathrm{U}$ & NG & NG \\
\hline \multicolumn{10}{|l|}{ Bulbar onset } \\
\hline 11 & $\mathrm{~F}$ & 55 & + & + & 85 & 77 & SP & $\mathrm{N}$ & NG \\
\hline 12 & M & 69 & + & + & 54 & 14 & SP & NG & NG \\
\hline 13 & $\mathrm{~F}$ & 75 & + & + & 69 & 72 & $\mathrm{U}$ & NG & NG \\
\hline 14 & $M$ & 68 & + & + & 87 & 38 & SP & NG & NG \\
\hline 15 & M & 62 & - & + & 85 & 7 & $\mathrm{U}$ & NG & NG \\
\hline 16 & $\mathrm{~F}$ & 72 & + & + & 88 & 63 & SP & $\mathrm{N}$ & NG \\
\hline 17 & M & 57 & + & + & 46 & 23 & SP & NG & NG \\
\hline 18 & M & 52 & + & + & 52 & 30 & $\mathrm{U}$ & NG & NG \\
\hline
\end{tabular}

UMNS = Upper motor neuron signs; BS = bulbar signs; SA = spontaneous activity (fibrillations, or fasciculations, or repetitive discharges); $\mathrm{SP}=$ definitely present; $\mathrm{NG}=$ neurogenic; $\mathrm{N}=$ normal.

needle was fixed manually by reining. To assess spontaneous activity, subjects were told to attempt retracting and relaxing the tongue. To record MUAPs, subjects were told to protrude the tongue until single MUAPs could be differentiated. In each subject, 20 different MUAPs were recorded. Between two sites the needle was withdrawn for at least $2-3 \mathrm{~mm}$. To record interference patterns, subjects were told to increase force continuously from zero to maximum within about 10 s, by pressing the tongue to the left against the investigator's index finger inside the mouth. In each subject, interference patterns were recorded at 10 different sites. Between two different sites, the needle was withdrawn for at least $2-3 \mathrm{~mm} .{ }^{4}$

Before or after the EMGs the patients' bulbar function was assessed by a logopedist, applying the Frenchay aphasia test. ${ }^{5}$ Motor abilities were assessed by means of the Norris score $^{6}$ after the EMGs.

INSTRUMENTATION

The EMGs were recorded via standard concentric needle electrodes (length 25 or $50 \mathrm{~mm}$, recording surface: $0.07 \mathrm{~mm}^{2}$ ) by means of a commercially available EMG recorder (Counterpoint, Dantec, Skovlunde, Denmark). To record MUAPs the sensitivity was set at 100 $\mu \mathrm{V}$, the filters between $20 \mathrm{~Hz}$ and $10 \mathrm{kHz}$, and the sweep speed at $5 \mathrm{~ms} /$ division. Ongoing MUAPs were selected by an amplitude trigger at a delay of $40 \%$. Among the recorded MUAPs, the five most similar potentials were averaged and MUAP indices assessed manually on line. MUAP duration was assessed on the most uncontaminated of the MUAPs. To

Table 2 Mean (2SD) of MUAP and peak ratio indices in 30 healthy subjects

\begin{tabular}{lc}
\hline Index & Mean (2SD) \\
\hline MUAP duration $(\mathrm{ms})$ & $6.6(1.5)$ \\
MUAP amplitude $(\mu \mathrm{V})$ & $224 \quad(97.4)$ \\
Peak ratio $(1 / \mu \mathrm{Vs})$ & $1.68(0.56)$ \\
Turns/s $(1 / \mathrm{s})$ & $732(303.9)$ \\
Amplitude/turn $(\mu \mathrm{V})$ & $446(180.3)$ \\
Time intervals $0-1.5 \mathrm{~ms}(\log$ values) & $2.62(0.34)$ \\
Time intervals $1.5-5 \mathrm{~ms}(\log$ values) & $2.31(0.14)$ \\
Time intervals $5-20 \mathrm{~ms}(\log$ values) & $1.01(0.50)$ \\
\hline
\end{tabular}

record interference patterns the sensitivity was set at $1 \mathrm{mV}$, the filters between $20 \mathrm{~Hz}$ and 10 $\mathrm{kHz}$, and the sampling frequency at $100 \mathrm{kHz}$. Interference patterns were evaluated by means of the peak ratio program (Department of Clinical Neurophysiology, University of Copenhagen), visualised on an external VGA screen.

\section{STATISTICS}

Evaluated indices were age, sex, disease duration, Frenchay aphasia test, Norris score, MUAP duration, MUAP amplitude, the rate of polyphasia (relative number of MUAPs with $>4$ phases), the peak ratio, the turns/second $(\mathrm{T} / \mathrm{s})$ at peak ratio, the amplitude/turn $(\mathrm{A} / \mathrm{T})$ at peak ratio, and the logarithms of the time intervals $0-1.5 \mathrm{~ms}$ (TI1), $1.5-5 \mathrm{~ms}$ (TI2), and 5-20 ms (TI3). Data distribution was established with the Kolmogorov-Smirnov test. Differences between group means were calculated with Student's $t$ test for independent samples (normal distribution) and with the MannWhitney $U$ test (non-normal distribution). Correlations between any of the indices were expressed as Pearson's correlation coefficients. Individual amyotrophic lateral sclerosis values were compared with control values using means (2SD). The sensitivity was calculated as (true positives/(true positives + false negatives) $) \times 100$. The specificity was calculated as (true negatives/(/true negatives + false positives $) \times 100 .^{7} \dagger$

\section{Results}

CONTROLS

Figure 1 shows the MUAPs from a healthy subject. MUAP amplitude and the $\mathrm{A} / \mathrm{T}$ were significantly $(\mathrm{P}=0.008, \mathrm{P}=0.012)$ higher in men than in women. As all other indices were not significantly different between the sexes, data were combined. None of the EMG indices was age dependent. Except for the rate of polyphasia, all other EMG indices were normally distributed. Table 1 shows the normal mean MUAP and peak ratio indices. The highest individual rate of polyphasia $(20 \%)$ was taken as an upper reference limit. 

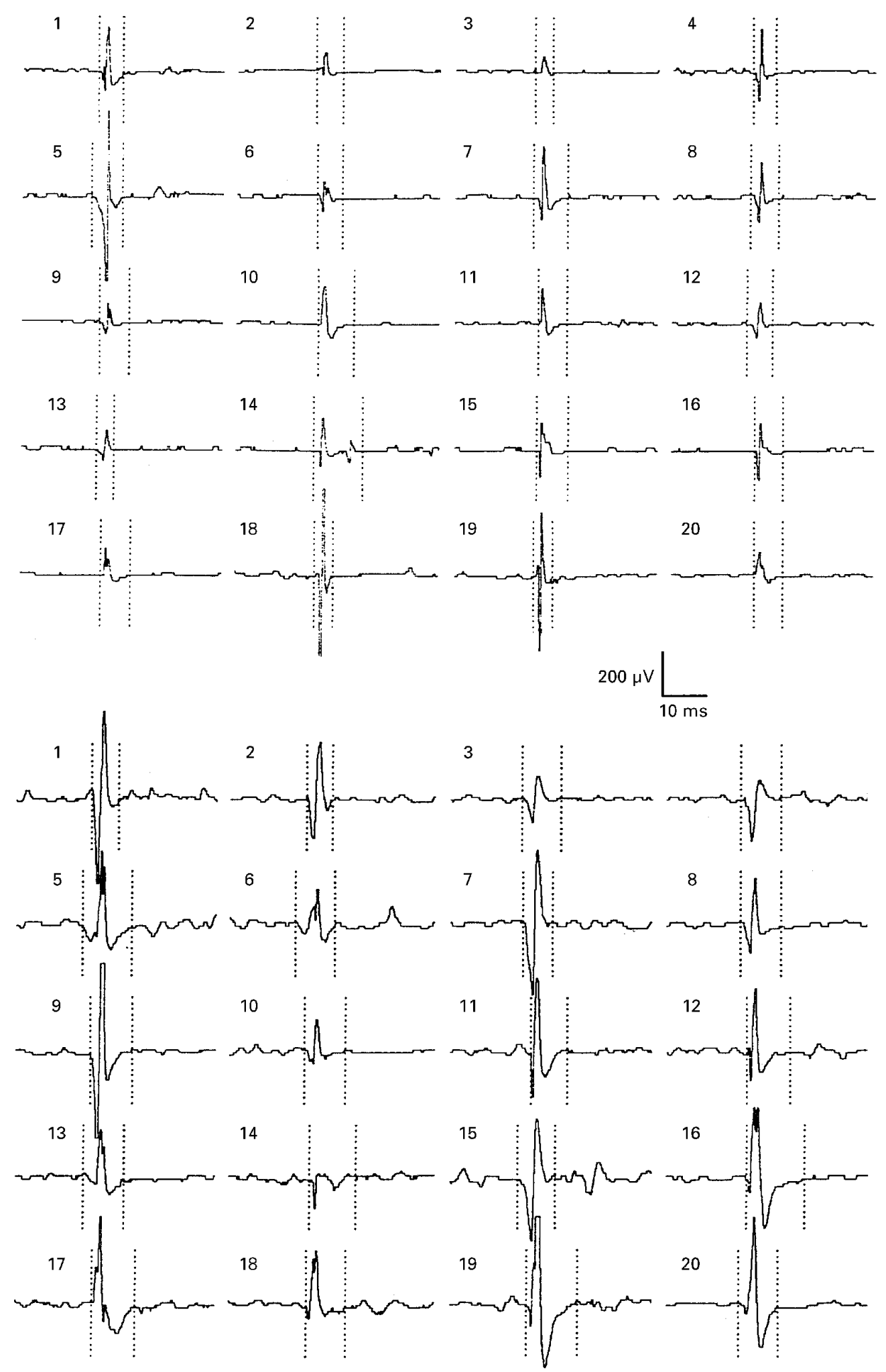

Figure 1 MUAPs from the right genioglossus muscle of a healthy subject (upper panel) and a patient with bulbar onset amyotrophic lateral sclerosis (lower panel). Markers for the MUAP duration were set on the most uncontaminated of the five averaged MUAPs.

PATIENTS

Upper motor neuron signs were found in $90 \%$ of the limb onset and $88 \%$ of the bulbar onset patients with amyotrophic lateral sclerosis (table 1). At the time of the examination $50 \%$ of the limb onset and $100 \%$ of the bulbar onset patients showed bulbar deficits (table 1).
In limb onset patients EMG accurately demonstrated fibrillations in five, fasciculations in three, and repetitive discharges in one. In patients with bulbar onset amyotrophic lateral sclerosis EMG accurately demonstrated fibrillations in five, fasciculations in four, and repetitive discharges in three. For fibrillations, 

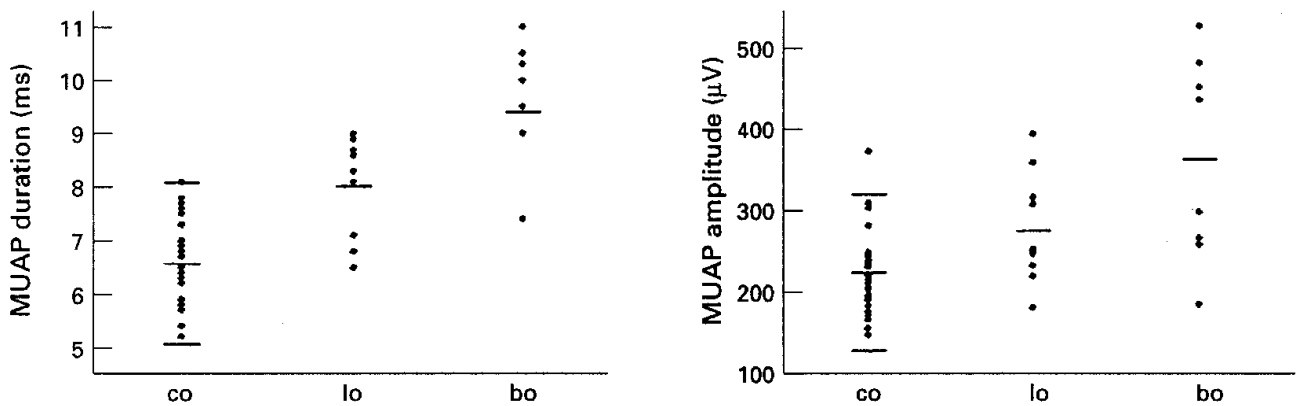

Figure 2 Individual mean MUAP duration and amplitude in healthy subjects (co) and patients with limb onset (lo) and bulbar onset (bo) amyotrophic lateral sclerosis (closed circles). Group means and reference limits (2SD) are given (horizontal bars).
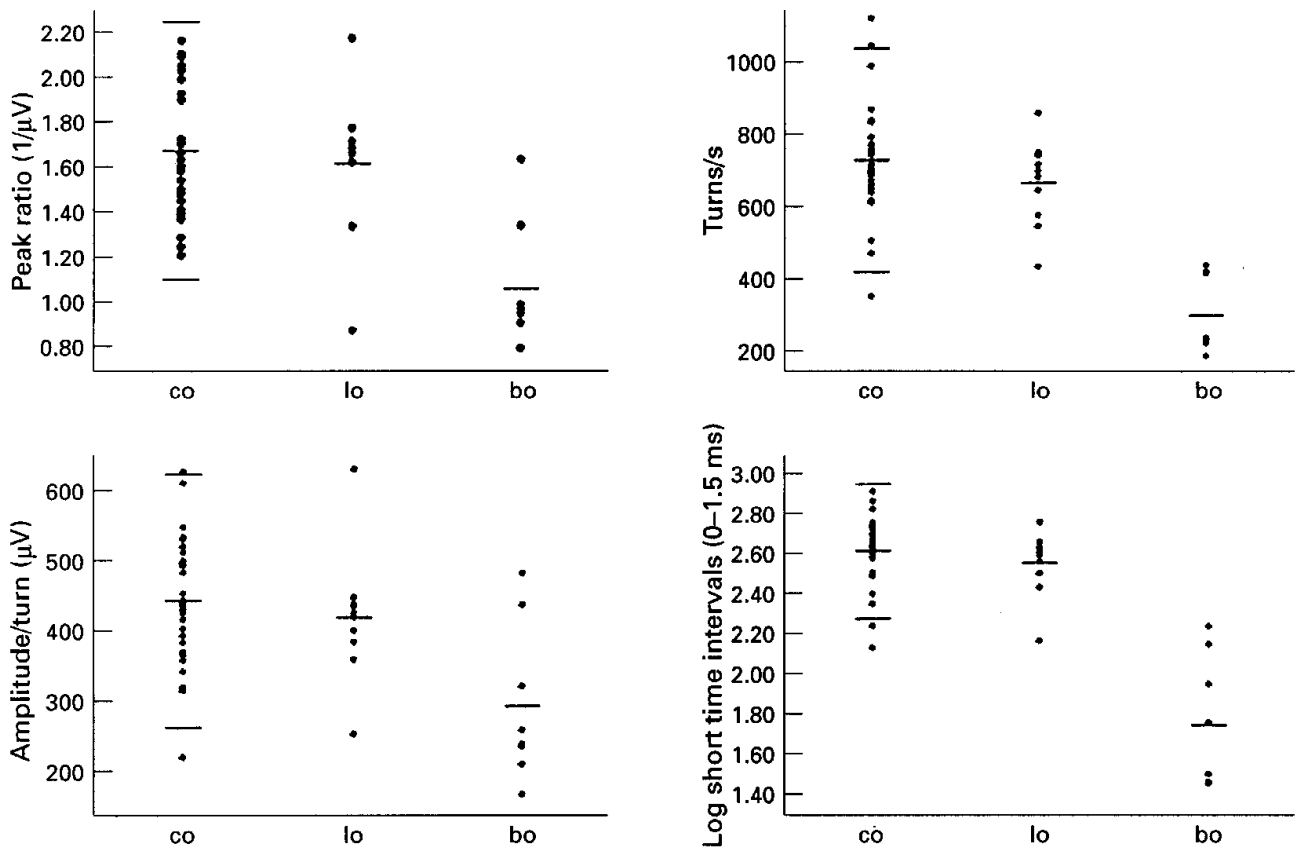

Figure 3 Individual mean peak ratio, turns/second, amplitude/turn, and short time intervals in healthy subjects (co) and patients with limb onset (lo) and bulbar onset (bo) amyotrophic lateral sclerosis (closed circles). Group means and reference limits (2SD) are given (horizontal bars).

fasciculations, and repetitive discharges together, spontaneous activity was found in $60 \%$ of the patients with limb onset and in $63 \%$ of the patients with bulbar onset. In the remaining patients spontaneous activity could not be assessed accurately.

Figure 1 shows the MUAPs from a patient with bulbar onset amyotrophic lateral sclerosis. MUAP duration was significantly increased in limb $(\mathrm{P}=0.0001)$ and bulbar onset amyotrophic lateral sclerosis $(\mathrm{P}=0.0001)$. MUAP amplitude was significantly increased in limb $(P=0.013)$ and bulbar onset amyotrophic lateral sclerosis $(\mathrm{P}=0.017)$ as well. MUAP duration was significantly increased $(P=0.02)$ in bulbar compared with limb onset (figure 2). The rate of polyphasia was not significantly different between the groups. The rate of polyphasia was increased in one limb onset and one bulbar onset patient each.

In limb onset amyotrophic lateral sclerosis none of the peak ratio indices was significantly different from controls. In bulbar onset amyotrophic lateral sclerosis, the peak ratio, $\mathrm{T} / \mathrm{S}$, $\mathrm{A} / \mathrm{T}, \mathrm{TI} 1$, and TI2 were significantly decreased compared with both controls $(\mathrm{P}=0.0001$,
$\mathrm{P}=0.0001, \mathrm{P}=0.0001, \mathrm{P}=0.0001, \mathrm{P}=0.013$ ) and patients with limb onset amyotrophic lateral sclerosis $\quad(\mathrm{P}=0.002, \quad \mathrm{P}=0.0001$, $\mathrm{P}=0.019, \mathrm{P}=0.0001, \mathrm{P}=0.008$ ) (fig 3). Compared with controls and patients with limb onset amyotrophic lateral sclerosis, the TI3 was significantly increased $(\mathrm{P}=0.0001, \mathrm{P}=0.0001)$ in bulbar onset amyotrophic lateral sclerosis.

Figure 4 gives the number of patients with abnormal EMG indices. Compared with bulbar onset amyotrophic lateral sclerosis, peak ratio indices were hardly abnormal in limb onset amyotrophic lateral sclerosis. For MUAP indices, there was hardly any difference between the two groups of patients.

MUAP analysis was assessed as neurogenic if MUAP duration or MUAP amplitude were increased. Peak ratio interference pattern analysis was assessed as neurogenic if the peak ratio, or the $\mathrm{T} / \mathrm{S}$, or the TI1 were decreased or the TI 3 was increased. Figure 5 gives the sensitivity and specificity of the MUAP analysis and the peak ratio interference pattern analysis. In limb onset amyotrophic lateral sclerosis the sensitivity of the MUAP analysis was higher than that of the peak ratio interference pattern 

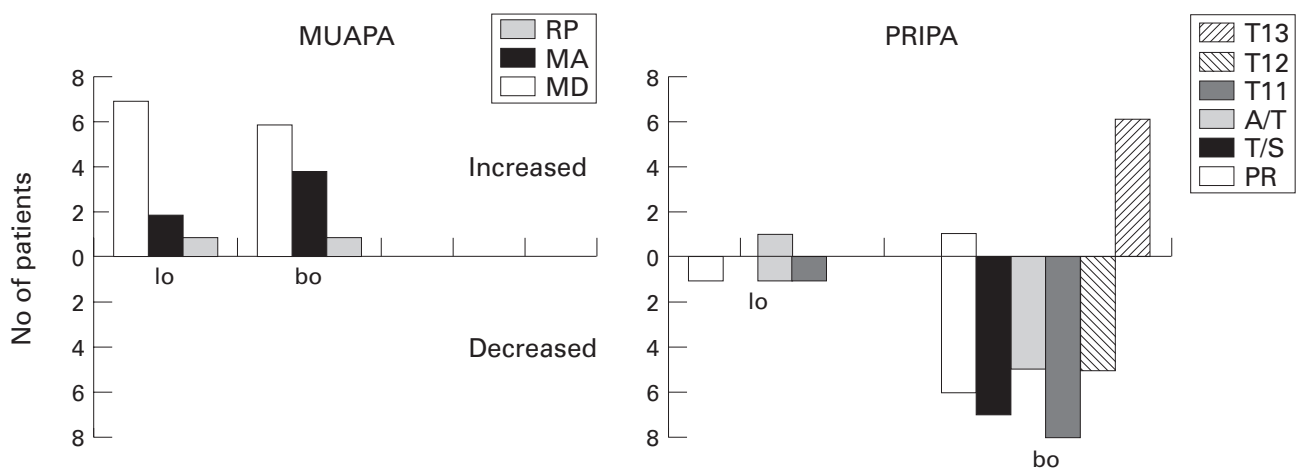

Figure 4 Number of limb onset (lo) and bulbar onset (bo) patients with amyotrophic lateral sclerosis with MUAP (left panel) and peak ratio indices (right panel) above (increased) or below (decreased) its corresponding reference limits. $R P=$ rate of polyphasia; $M D=M U A P$ duration; $M A=M U A P$ amplitude; $P R=$ peak ratio; $T S=$ turns/second; $A T=$ amplitude/turn; $T I 1-3=$ time intervals; $M U A P A=M U A P$ analysis; $P R I P A=$ peak ratio interference pattern analysis.

analysis and vice versa in bulbar onset amyotrophic lateral sclerosis. Peak ratio interference pattern analysis discriminated better between limb and bulbar onset amyotrophic lateral sclerosis than MUAP analysis (fig 5).

The mean Norris score was $69 \%$ in limb and $71 \%$ in bulbar onset amyotrophic lateral sclerosis $(\mathrm{P}=0.798)$. The mean Frenchay aphasia test was $91 \%$ in limb and $41 \%$ in bulbar onset amyotrophic lateral sclerosis $(\mathrm{P}=0.001)$. The Frenchay aphasia test was normal (>85\%) in $90 \%$ of limb onset and in none of the bulbar onset patients. MUAP and peak ratio interference pattern analysis were neurogenic in $60 \%$ and $20 \%$ of the limb onset patients with a normal Frenchay aphasia test. With increasing Frenchay aphasia test, MUAP duration and TI3 decreased $(r=-0.757$, $\mathrm{P}<0.05 ; r=-0.823, \mathrm{P}<0.05)$ and TI1 increased $(r=0.713, \mathrm{P}<0.05)$ in bulbar onset amyotrophic lateral sclerosis. The Norris score was negatively correlated $(r=-0.724, \mathrm{P}<0.05)$ with MUAP duration in bulbar onset amyotrophic lateral sclerosis.

\section{Discussion}

This study has shown that MUAP analysis and peak ratio interference pattern analysis of the tongue are different between healthy subjects and patients with limb onset or bulbar onset amyotrophic lateral sclerosis. The sensitivity of the MUAP analysis was higher than that of the peak ratio interference pattern analysis in limb

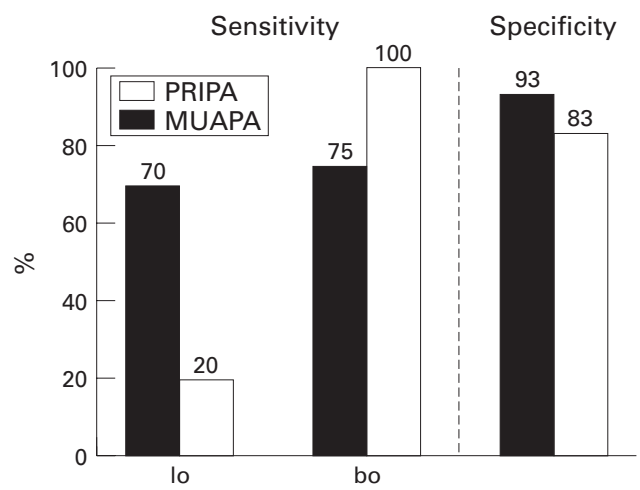

Figure 5 Sensitivity and specificity of MUAP analysis (MUAPA) and peak ratio interference pattern analysis (PRIPA) of the tongue in patients with limb onset (lo) and bulbar onset (bo) amyotrophic lateral sclerosis. onset amyotrophic lateral sclerosis and vice versa in bulbar onset amyotrophic lateral sclerosis. Concerning subclinical involvement of the tongue in the neurogenic process, the sensitivity of the MUAP analysis was higher than that of the peak ratio interference pattern analysis.

Although needle EMG of the tongue is said to be helpful in the diagnosis of various neurological disorders, ${ }^{8-12}$ it has been carried out only in a few isolated cases and rarely in a systematic investigation on a group of subjects. The following reasons might be responsible for the rare application of the needle EMG to the tongue: (1) needle EMG is thought to cause discomfort to the patient, (2) relaxation of the tongue is difficult for many subjects, (3) reference values for MUAP and peak ratio indices are limited, (4) the diagnostic value of the needle EMG of the tongue is unknown, and (5) some electrophysiologists may have an aversion against the insertion of a needle into such a sensitive organ.

Objections against needle EMG of the tongue can be easily overcome. Discomfort to the patient can be avoided if the tongue is sufficiently anaesthetised before needle insertion. Sufficient tongue relaxation can be reached by motivation and explanation. Reference limits for the MUAP duration, given by Buchthal and Jüde et al, are similar to those of the present investigation. Nevertheless, every electrophysiological laboratory should acquire its own reference limits, because of the different recording conditions. ${ }^{1}$ The lack of age dependency in the present and earlier investigations might be due to the selection bias, the recording conditions, and the speculative missing enlargement of tongue motor units with age. ${ }^{12}$ Reference limits for peak ratio interference pattern analysis indices have not been reported before. Concerning the diagnostic value, this study has shown that needle EMG of the tongue is helpful to detect subclinical and confirm clinical involvement of the tongue in patients with amyotrophic lateral sclerosis. Because MUAP duration and amplitude are small in healthy subjects, the diagnostic relevance for myopathies remains questionable. Aversion against needle insertion into the tongue can be overcome by sufficient anaesthe- 
sia and by routine. Furthermore, less invasive approaches to look for tongue involvement in amyotrophic lateral sclerosis can be applied percutaneously through the floor of the mouth.

The low sensitivity of the peak ratio interference pattern analysis in limb onset amyotrophic lateral sclerosis can be explained if only low threshold motor units in early limb onset amyotrophic lateral sclerosis are affected. These low threshold motor units seem to be more accurately registered with MUAP analysis than with peak ratio interference pattern analysis. Possibly, force was increased too rapidly, especially at the beginning of the contraction during the automatic EMG.

With needle EMG of the tongue, spontaneous activity is difficult to assess. This might be due to the similarity between fibrillation and fasciculation potentials and true MUAPs of the tongue and the difficulty in relaxing the tongue. Furthermore, pathological spontaneous activity is a non-specific finding in neuromuscular disorders and its general assessment does not seem to improve the diagnostic yield of the method. Primarily repetitive discharges can be accurately assessed with needle EMG of the tongue. The low rate of polyphasia in the present investigation can be explained with a presumed reduction of the tongue's reinnervation capacity and the selection of MUAPs during the recordings. As a pathological rate of polyphasia is a non-specific finding as well, its diagnostic value is of minor importance. Despite the examiner dependency, ${ }^{7}$ the most appropriate indices to detect a neurogenic lesion of the tongue are the MUAP duration and amplitude. MUAP duration correlates well with the Norris score and with the Frenchay aphasia test. Among the peak ratio indices, only TI1 and TI2 correlated with the Frenchay aphasia test. The missing correlation of the other peak ratio indices with clinical aspects can be explained with an inappropriate muscle contraction and the presumed preferential affection of low threshold motor units in amyotrophic lateral sclerosis. Some of the recruitment abnormalities described can be attributed to upper motor neuron involvement as well.

Limitations of the study were that MUAPs, hardly disturbed by background activity, were preferentially selected and that the tongue contraction for the peak ratio interference pattern analysis is not easy to standardise and depends on the size and shape of the organ.
It is concluded that both MUAP and peak ratio interference pattern analysis can detect neurogenic lesions in the tongue of patients with amyotrophic lateral sclerosis. MUAP analysis has higher sensitivity than peak ratio interference pattern analysis in detecting subclinical neurogenic lesions, whereas peak ratio interference pattern analysis discriminates better between limb and bulbar onset amyotrophic lateral sclerosis than MUAP analysis. On the whole, needle EMG of the tongue is a valuable, supplementary electrophysiological device to assess the clinical and subclinical involvement of the tongue in patients with amyotrophic lateral sclerosis.

We are grateful to Professor W Grisold for acquainting us with ique of conventional tongue EMG

1 Buchthal F. Einführung in die Elektromyographie. München: Urban und Schwarzenberg 1958.

2 Jüde HD, Drechsler F, Neuhauser B. Elementare elektromyographische Analyse und Bewegungsmuster des M. myoglossus. Dtsch Zahnärztl Z 1975;30:457-61.

3 World federation of neurology research group on neuromuscular diseases. Subcommittee on motor neuron diseases/ amyotrophic lateral sclerosis of the world federation of neurology research group on neuromuscular diseases and neurology research group on neuromuscular diseases and the El Escorial "clinical limits of amyotrophic lateral sclerosis" workshop contributors. El Escorial world federa-
tion of neurology criteria for the diagnosis of amyotrophic tion of neurology criteria for the diagnosis of
lateral sclerosis. 7 Neurol Sci 1994;124:96-107.

lateral sclerosis. F Neurol Sci 1994;124:96-107.
4 Liguori R, Dahl K, Fuglsang-Frederiksen A. Turnsamplitude analysis of the electromyographic recruitment pattern disregarding force measurement. I. Method and reference values in healthy subjects. Muscle Nerve 1992;15: $1314-8$

5 Enderby P, Crow E. Frenchay aphasia screening test: validity and comparability. Disabil Rehabil 1996;18:238-40.

6 Norris FH, Calanchini PR, Fallat RJ, Panchari S, Jewett B. The administration of Guanidine in amyotrophic lateral sclerosis. Neurology 1974;24:721-8.

7 Nirkko AC, Rösler KM, Hess CW. Sensitivity and specificity of needle electromyography: a prospective study comparing automated interference pattern analysis with single motor unit potential analysis. Electroencephalogr Clin Neurophysiol 1995;97:1-10

8 Finsterer J, Wogritsch C, Pokieser P, Vesely M, Ulrich W, Grisold W, Mamoli B. Light chain myeloma with oro-pharyngeal amyloidosis presenting as bulbar paralysis. 7 Neurol Sci 1997;147:205-8.

9 Kang D-X, Fan D-S. The electrophysiological study of differential diagnosis between amyotrophic lateral sclerosis and cervical spondylotic myelopathy. Electromyogr Clin Neurophysiol 1995;35:231-8.

10 Praud JP, D'Allest AM, Delaperche MF, Bobin S, Gaultier C. Diaphragmatic and genioglossus electromyographic activity at the onset and at the end of obstructive apnea in children with obstructive sleep apnea syndrome. Pediatr Res 1988;23:1-4.

11 Shimizu T, Miyamoto K, Hayashi H, Nagashima T, Hirose H, Tanabe H. Congenital facioscapulohumeral muscular dystrophy associated with tongue atrophy and sensodystrophy associated with tongue atrophy and sensoryneural

12 Tsuda N, Yamamoto K, Fukusako T, Morimatsu M. A case of unilateral lingual atrophy and ipsilateral muscular atrophy supplied by trigeminal nerve - in relation to progressive facial hemiatrophy. Rinsho Shinkeigaku 1991; 31:1007-9. 